\title{
The CESuRa System: Allowing Changes in a Rail Track Profile While Maintaining Full Vertical Support
}

\author{
Andy Foan ${ }^{1} \cdot$ Travis Thonstad $^{2} \cdot$ John F. Stanton ${ }^{2}$
}

Received: 26 August 2018/Revised: 19 October 2018/Accepted: 27 October 2018/Published online: 9 November 2018

(C) The Author(s) 2018

\begin{abstract}
A light rail passenger transportation system is being built in Seattle, WA, USA, that traverses Lake Washington over an existing pontoon bridge. The water level in Lake Washington changes throughout the year. This causes rotations of the transition spans, which are needed at each end of the bridge to carry traffic between the fixed, land-based structure, and the floating structure. This paper explains a novel method, the Curved Element Supported Rail (CESuRa) system, that provides rails with the ability to undergo joint rotations at the ends of the transitions spans without risk of damage, while maintaining full vertical support of the track across the joint. The geometric characteristics on which it depends for proper operation, considerations that must be accounted for when using the system for other applications in the future, and an overview of the implementation of the system on the floating bridge are discussed.
\end{abstract}

Keywords Light rail transit - Floating bridges - Pontoon bridges $\cdot$ Expansion joints $\cdot$ Rail hinge $\cdot$ Track bridge

Travis Thonstad

thonstat@uw.edu

Andy Foan

sandc@andyfoan.co.uk

John F. Stanton

stanton@uw.edu

1 Andy Foan Limited, 15 Bloomsbury Drive, Nuthall, Nottingham NG16 1RJ, UK

2 Department of Civil and Environmental Engineering, University of Washington, 201 More Hall,

Box 352700, Seattle, WA 98195-2700, USA

Editor: Qi Li.

\section{Introduction}

A light rail passenger transportation system is being built in the Puget Sound Region in Seattle, WA, USA. Some sections are already complete, others are under construction, and others still are in the design phase. One section will cross Lake Washington on the existing Homer M. Hadley Memorial Bridge, a floating bridge which is named after the engineer who first proposed the concept of crossing the lake using a bridge made from concrete pontoons. The lake is sufficiently deep, and the underlying soil is sufficiently poor, so that the cost of a fixed bridge, such as suspension span, would have been prohibitive.

The water level in Lake Washington changes throughout the year and is controlled by adjustments to a weir system through which the fresh lake water flows out to the salt water of Puget Sound. The variable lake level means that a transition span is needed at each end of the bridge to carry traffic between the fixed, land-based structure and the floating structure. The transition span in the bridge, shown in Fig. 1, plays much the same role as a ship's gang-plank in harbor; it pivots freely at each end to accommodate the rise and fall of the floating structure.

The transition span of the floating bridge is subject to three displacement-induced rotations (Fig. 2), and one relative displacement, of the transition span relative to the fixed or floating segments. The largest rotation at the bridge joints is caused by the variation in lake water level. The vertical displacement of the floating portion of the bridge caused by lake level changes, shown in Fig. 2a, causes a "pitch" motion that results in rotations about a horizontal transverse axis at each end of the transition span.

In addition to the larger, planned, changes in lake level, the floating part of the bridge is in constant motion under the action of wind, traffic and thermal loading. The bridge 


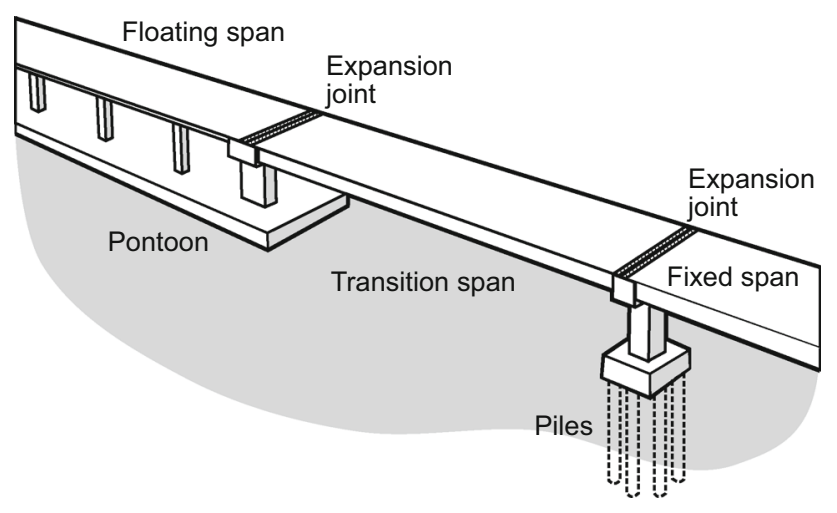

Fig. 1 Fixed, transition and floating spans of the Homer M. Hadley Bridge

(a)

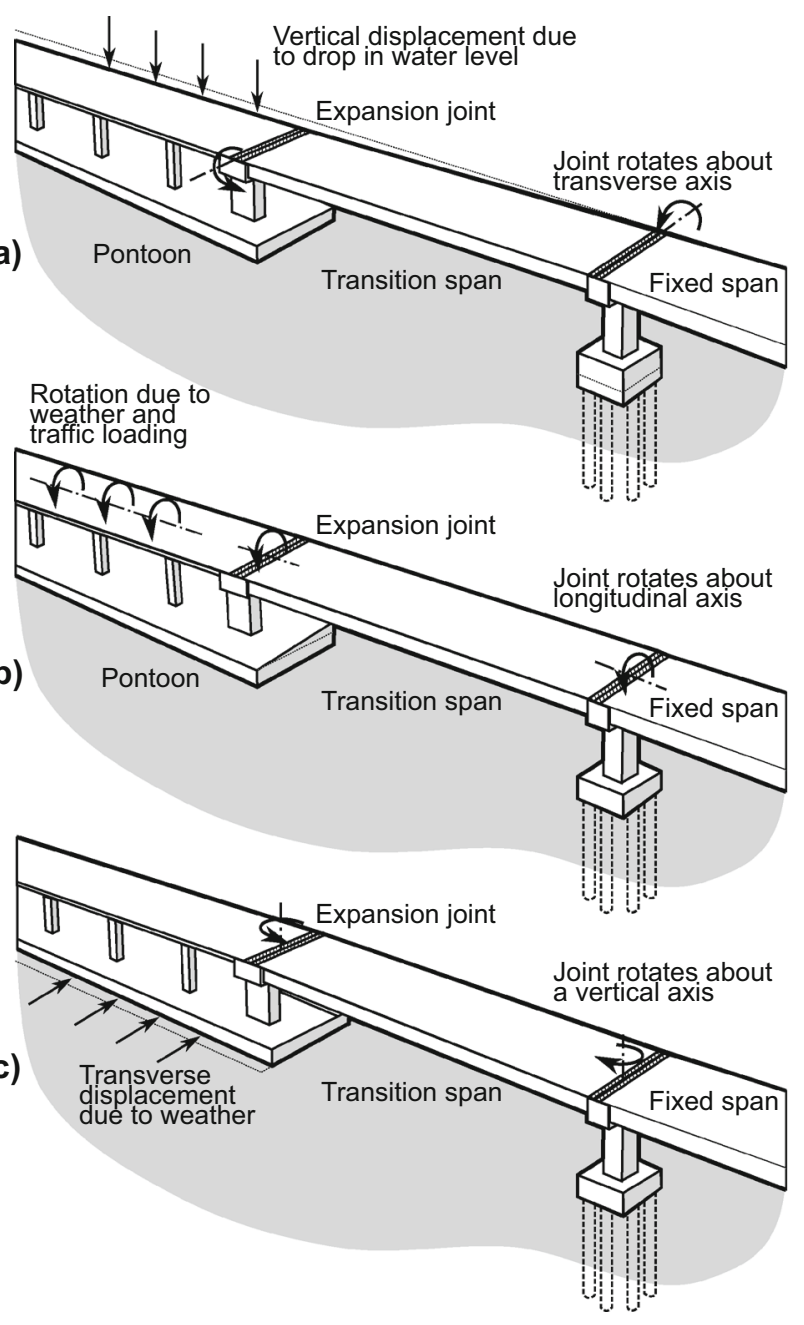

Fig. 2 Bridge movements and corresponding joint rotations, (a) pitch, (b) roll and (c) yaw

runs approximately east-west, and the track is to be placed on the south edge. When a train enters the floating section of the bridge, its self-weight and the associated dynamic forces are expected to cause a twisting, or "roll" motion
(Fig. 2b), that results in $229 \mathrm{~mm}$ (9 in.) downward displacement on the loaded, south, side of the floating bridge [1]. Rotation about the vertical axis, or "yaw," occurs if the floating span moves laterally, perhaps in response to wind forces, as shown in Fig. 2c.

In addition to rotations, translations are in general possible in all three degrees of freedom. Horizontal and vertical displacements, which may be thought of as shear displacements, are not possible because the floating and transition spans rest on a common cross-beam. However, relative longitudinal motion, or "surge," may occur as a result of thermal expansion of the rails or gross movements of the bridge decks.

These motions are of little consequence for road traffic traveling on pneumatic tires, because the rotations of the transition span are small (less than $3^{\circ}$ ). However, they pose problems for a fixed rail system, which cannot undergo, without yielding, a concentrated rotation at the pivot points. Even if a pin could be introduced, the resulting concentrated rotation would cause impacts that would prove unacceptable to the riders in terms of comfort and safety [2,3], and would risk causing damage to the mechanism. It was therefore necessary to develop a system that would spread the rotations over a sufficiently long length of rail that the resulting curvature induced stresses would be acceptably small, but that would at the same time provide vertical support for the track at every tie.

These two requirements appear to be mutually exclusive, as illustrated for a pitch rotation in Fig. 3. To keep the bending stress low, the rail must be allowed to bend over a long free length, but such a length implies lack of vertical support, in which case the track would be too flexible and weak to support a vehicle crossing it (Fig. 3a). By contrast, closely spaced supports imply a short length between them in which the concentrated rotation must occur (Fig. 3b). Neither behavior is acceptable.

These motions are not unique to floating structures; however, the frequency, combination, and magnitude of the expected rotations are. Sharp changes in alignment can occur at transitions between direct fastened and ballasted

(a)

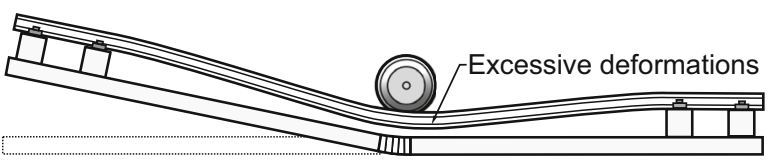

(b)

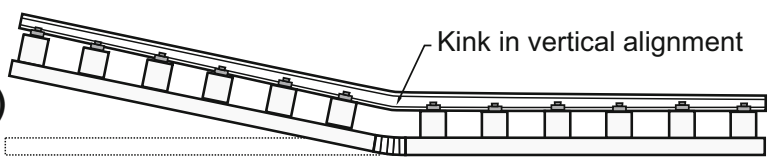

Fig. 3 Track bending and support requirements, (a) rail is allowed to bend over a long free length, (b) rail is fully supported across the joint 
track, due to settlement of the track-bed. Solutions have been developed to improve ride quality and reduce maintenance demands at these transitions [4-7]; however, these solutions are either remedial or are implemented in the design phase to mitigate track-bed settlement and avoid the issue. Hinged joints can often be found at the transitions between bridges and abutments, and solutions have also been developed that can accommodate limited amounts of rotation at these transitions [8-11]. For example, the SkyTrain system in Vancouver, BC, Canada, encounters a combination of surge $\left(13.1 \mathrm{in}\right.$.) and pitch $\left(0.75^{\circ}\right)$ at the span abutments, as it traverses the cable-stayed bridge crossing the Fraser River [8]. These motions, caused by temperature and light rail vehicle forces, are accommodated by a standard expansion joint at the abutment and specialized fasteners near the transition that include elastomeric springs. The springs allow the rails to bend over the transition section while still providing sufficient support to maintain ride quality [8].

Where rotations are beyond the capacity of the rail to simply bend between fasteners, a structural transition to divide the bend over multiple portions of track can be used [9-11]. The Tagus River Bridge in Lisbon, Portugal, is an example. Thermal expansion of the continuous steel truss causes large surge movements $(1.5 \mathrm{~m}$ or $60 \mathrm{in}$.) and pitch rotations $\left(3.43^{\circ}\right)$ at the bridge abutments [9]. The large surge displacements at the abutments are accommodated by a sliding box girder that straddles the bridge joint. The use of the transition girder divides the pitch rotation in two, half on either end of the girder. The resulting rotation demands are accommodated by fasteners connected to ties through neoprene shims [9].

The concept of a structural transition could be extended to include a linkage consisting of multiple beams [e.g., 10-11], as is shown schematically in Fig. 4. In the figure, three beams are connected by pins near the end of the center beam; the end beams are free to slide longitudinally

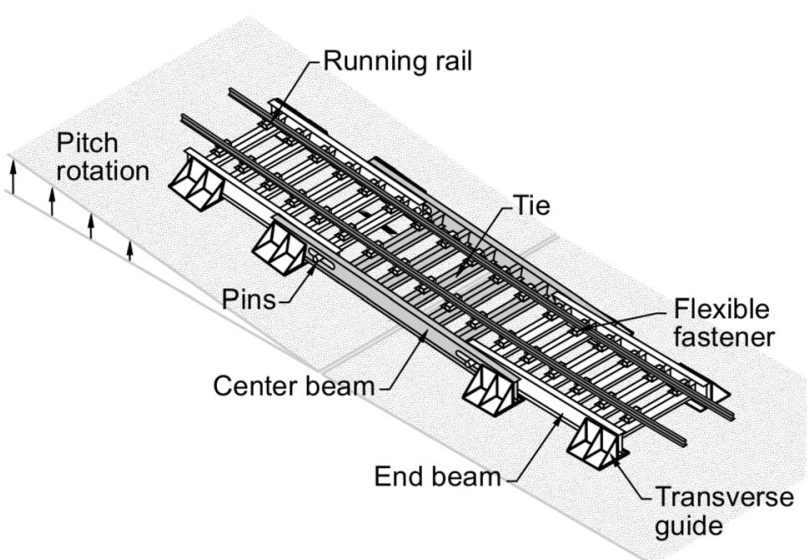

Fig. 4 Three-beam system for accommodating track but restrained in the transverse direction by guides. As the transition span rotates up or down, one end of the center beam rises or falls with it, and the pins between the beams force the end beams to rotate. The concentrated rotation is thereby divided into four components, at the ends of the linkage and between the connected beams. One such threebeam linkage is placed on either side of the track, and the ties span between the beams. The track is supported by the ties and attached with fasteners that provide some vertical flexibility to allow a reasonably smooth transition between the three straight line segments of the supporting beams. Because the linkage is located above the hinge in the bridge, some longitudinal movement is needed in the center beams, which could be slotted and free to slide relative to the end beams.

While this system provides a geometric solution, in practice its success would depend on the ability of the beams to provide stiff and stable support to the track. This would be available under pitch motions of the transition span. Under roll motions, the beams would also have to permit free torsional deformations as one side of the track rise and the other fall. In both cases, the action would have to be reliable over time in a hostile and wet environment. These are not conditions in which complex mechanical systems, subjected to heavy dynamic loads, thrive.

A new system has been developed [2, 11, 12], which provides a smooth continuous curve across a bridge transition, while providing continuous support to the rails across the bridge joint. It is called the "Curved Element Supported Rail," or CESuRa, system, and is shown schematically in Fig. 5. This paper addresses the concept, kinematics, and expected response of the CESuRa system. The intent of the paper is to describe the geometric design philosophy of the CESuRa system so that it could be used in future applications.

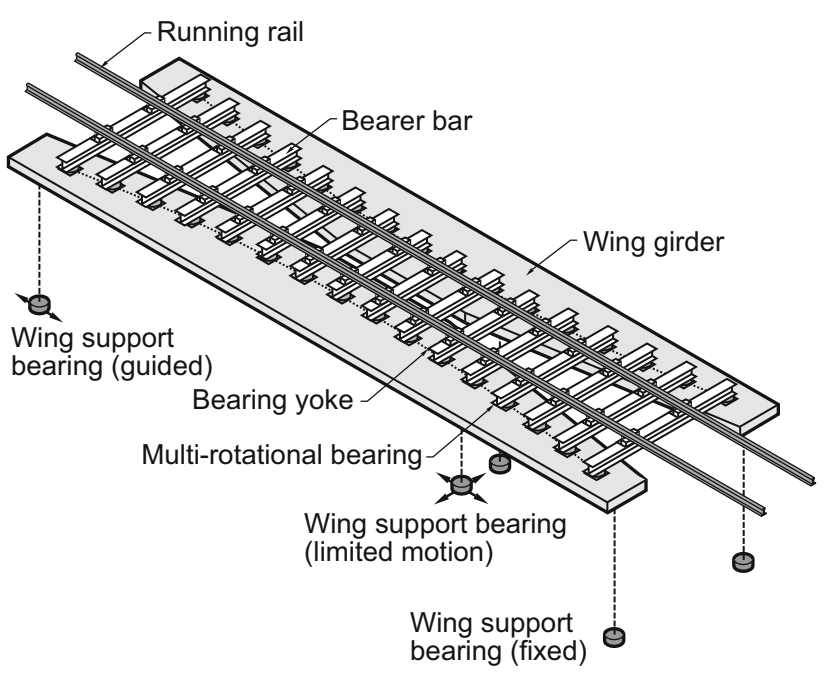

Fig. 5 Schematic of the CESuRa system 
A brief discussion on the implementation of this system for light rail transit over a floating bridge in Seattle, WA, USA, is also included. Additional information about the project and the development of the CESuRa system can be found in $[2,11,12]$. The benefits of the CESuRa system, in terms of rail stresses and vertical acceleration, and potential applications are summarized in [2].

The floating bridge project included evaluation of the CESuRa system using numerical simulations [13], preliminary proof-of-concept tests at the University of Washington [14, 15], and full-scale tests of the system under dynamic LRV loads [16, 17]. These studies showed that the system could accommodate joint rotations in excess of the project requirements with LRV travel at $88.5 \mathrm{kph}$ (55 $\mathrm{mph}$ ) [16]. Stresses in the rails and bearer bars were found to be less than $138 \mathrm{MPa}$ (20 ksi) under these conditions [14, 17], the lateral displacements under LRV loading were less than $6 \mathrm{~mm}$ (0.25 in.) [17], and the vertical and lateral accelerations were found to be well within acceptable limits $[13,16,17]$. These studies investigated one potential geometry of a CESuRa system and showed that it could meet the project requirements. In new applications, the system can be used to achieve similar levels of performance; however, the geometry of the system must be designed to meet the specific project requirements. How this should be done is explained in the following sections.

\section{CESuRa Concept}

The CESuRa concept is most easily understood in the context of pitch, which is also the motion that it was developed to address. Figure 6a shows the ends of the floating and transition spans in the neutral, or undeformed configuration. The spans are represented by planes, attached along hinge line DD'. In this position, the two planes are co-planar and, in the interests of simplicity, may here be treated as horizontal. Two additional, triangular planes, ABC and A'B'C', rest on the two rectangular ones symmetrically about the hinge line DD'. They are referred to here as "wings." On each wing is drawn an arc, or "yoke," along which multi-rotational bearings are placed. Those bearings support transverse beams, or "bearer bars," that in turn support the rails of the track, shown schematically in Fig. 5.

Figure $6 \mathrm{~b}$ shows the ends of the floating and transition spans in the sagging curve deformed configuration, i.e., when the lake level is below the neutral position. If the rectangular planes (the floating and transition spans) rotate relative to each other along hinge line DD', the ends B and $B$ ' of the wings will rise and the wings will rotate about their longitudinal edges, namely axes $\mathrm{AB}^{*}$ and $\mathrm{A}^{\prime} \mathrm{B}^{\prime} *$, in

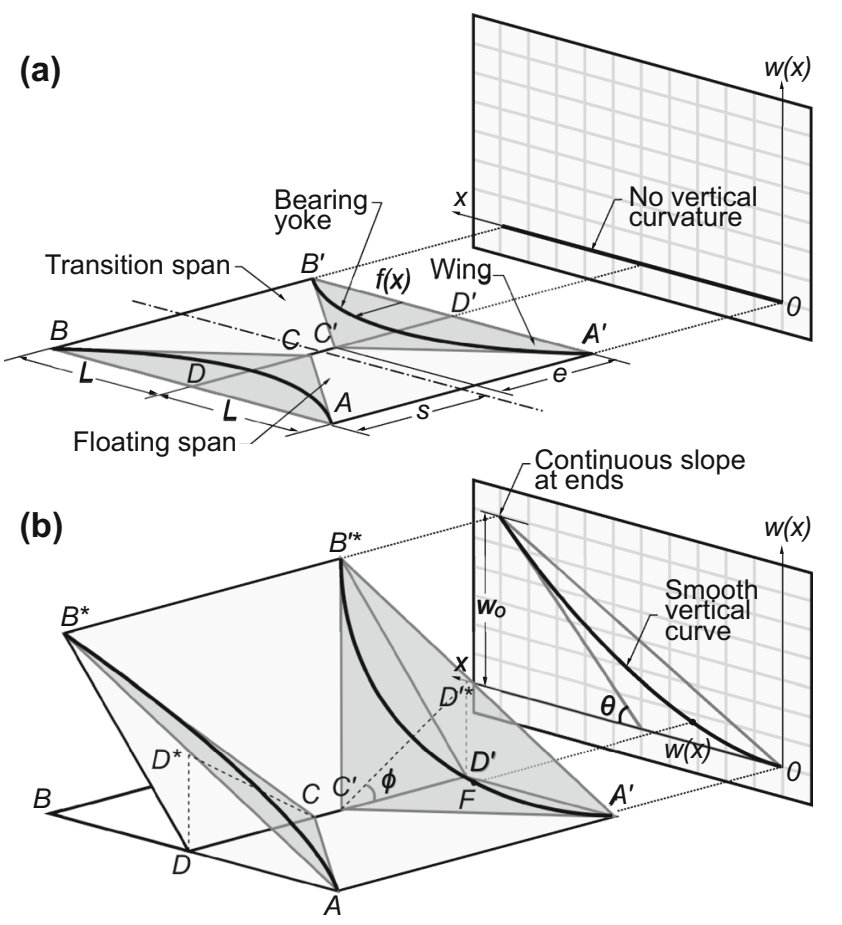

Fig. 6 Geometry for analysis of pitch motions, (a) undeformed configuration, (b) deformed configuration (rotations are greatly exaggerated)

order to maintain contact with the spans at points $C$ and $C^{\prime}$. As the wing $\mathrm{ABC}$ rotates, the arc will rotate about the chord line $\mathrm{AB}^{*}$ so that it is no longer in the horizontal plane. The bearings that are placed along the arc, and the bearer bars that rest on them, will therefore move vertically. The bearer bars are free to slide transversely on the bearings through a limited distance, and the rails that rest on them adopt a vertically curved shape equal to the vertical component of the arc on the rotated wing. The bearings can be placed at any spacing on the wing, so any degree of support can be provided to the rails while forcing them to go through the desired vertical curvature. If the wings and bearer bars were infinitely stiff, the rails would be supported rigidly at each bearer bar and the vertical curve of the track would be the one defined by the rotated arc. In practice, they will have some flexibility, so the shape of the track will differ slightly from the idealized shape computed in the following sections.

The wings must, at minimum, be supported at three locations, two at A and B and a third, "offset," wing support bearing along the line AC. In this configuration, the wing support bearings must primarily accommodate rotation, but also some translation. The need for translation is caused by the fact that paths $\mathrm{ADB}^{*}$ and $\mathrm{AD}^{*} \mathrm{~B} *$ in Fig. $6 \mathrm{~b}$ are not the same length. One wing support bearing (e.g., the one at $\mathrm{A}$ ) should be fixed against longitudinal and transverse displacements. The one at $\mathrm{B}^{*}$ should then be guided, 
permitting free longitudinal but not transverse displacement. The offset wing support bearing, along the line $\mathrm{AC}$, must be designed for rotation about the line $\mathrm{AC}$ and for some longitudinal and transverse displacement to accommodate possible yaw motions.

This use of 3-D rigid body motions is the basic concept underlying the CESuRa system. Unlike the systems described previously, it does not rely on flexible bearings to create a smooth vertical curve for the track. The geometry can be chosen to meet certain design objectives, and these are described in the next section.

\section{Geometric Requirements}

Design criteria are needed to select the geometry of the system. The most important are:

- Avoid sharp changes in slope in the rails in the event of pitch motion,

- Avoid sharp changes in lateral or twisting motions in the event of roll motion,

- Minimize vertical acceleration of the vehicle for any given travel speed,

- Accommodate other motions such as yaw rotation and surge displacement.

These criteria are addressed in the following sections.

\subsection{Pitch}

To satisfy the first criterion, the vertical profile of the rails must be continuous from the floating span to the CESuRa track bridge, and from the CESuRa track bridge to the transition span. Figure $6 \mathrm{~b}$ shows a system subjected to pitch motion, in which the CESuRa track bridge is placed symmetrically about the hinge joint between the floating and transition spans.

The dimensions of the elements are shown in Fig. 6a. The length of the wing, $\mathrm{ABC}$, is $2 L$, with a height along line $\mathrm{CD}$ of $e$. The perpendicular distance from the line $\mathrm{AB}$ to the system's centerline is $s$. The transverse coordinates of the bearing yoke in the horizontal plane are defined by: $y=f(x)$

where $f(x)$ is a curve to be selected by the designer. Now let the end of the wing that rests on the transition span (point B) rise through a distance $w_{0}$ to the position $\mathrm{B}^{*}$.

Point $\mathrm{D}$ rises to location $\mathrm{D}^{*}$, through a distance $w_{0} / 2$, so, in order for point $\mathrm{C}$ to remain in contact with the floating span, the wing must rotate about axis $\mathrm{AB}^{*}$ through an angle $\phi$ (assume for simplicity that $\phi \ll 1$ ), given by: $\phi \approx \sin \phi=\frac{w_{0}}{2 e}$

The total vertical displacement of a point along the arc consists of the upward displacement due to the pitch of the wing plus a downward component due to the rotation of the wing about axis $\mathrm{AB}^{*}$. The vertical displacement of the arc at a location $x$ along the horizontal plane is then given by:

$w(x)=\frac{w_{0} x}{2 L}-f(x) \phi=\frac{w_{0} x}{2 L}-\frac{w_{0}}{2 e} f(x)=\frac{w_{0}}{2}\left(\frac{x}{L}-\frac{f(x)}{e}\right)$

The slope of the rail is:

$\frac{\mathrm{d} w(x)}{\mathrm{d} x}=\frac{w_{0}}{2}\left(\frac{1}{L}-\frac{1}{e} \frac{\mathrm{d} f(x)}{\mathrm{d} x}\right)$

where $f(x)$ and its derivative can be taken with respect to the horizontal axis, because of the assumption of small angles, without loss of generality of the resulting relationship. For slope continuity at the ends of the track bridge, $\frac{\mathrm{d} w}{\mathrm{~d} x}$ must have the value 0 at $x=0$, and $w_{0} / \mathrm{L}$ at $x=2 L$. These conditions lead to the requirements below:

$\left.\frac{\mathrm{d} f(x)}{\mathrm{d} x}\right|_{x=0}=\frac{e}{L}$

$\left.\frac{\mathrm{d} f(x)}{\mathrm{d} x}\right|_{x=2 L}=-\frac{e}{L}$

Equations (5a) and (5b) provide a simple geometric relationship to ensure continuity in vertical slope in the rails. Their physical interpretation is that the line AC must be tangent to the bearing yoke on which the bearings are placed on the wing. The shape of the bearing yoke itself may be chosen arbitrarily by the designer provided that it satisfies Eqs. (5a) and (5b). For a symmetric hinge, simple candidate shapes include a circular arc and a parabola. For example, if a circle of radius, $r$, is chosen, the function $f(x)$ takes the form:

$f(x)=L \sqrt{\left(\frac{r}{L}\right)^{2}-\left(1-\frac{x}{L}\right)^{2}}-L \sqrt{\left(\frac{r}{L}\right)^{2}-1}$

For slope continuity at the ends of the track bridge, Eq. (5a) must be satisfied. Taking the derivative of $f(x)$ and evaluating it at zero leads to:

$\left.\frac{\mathrm{d} f(x)}{\mathrm{d} x}\right|_{x=0}=\frac{1}{\sqrt{\left(\frac{r}{L}\right)^{2}-1}}=\frac{e}{L}$

which can be rearranged to yield:

$\left(\frac{L}{e}\right)^{2}+1=\left(\frac{r}{L}\right)^{2}$ 
From Eq. (8), the radius of the circle, $r$, the length of the CESuRa system, $L$, and the location of the offset bearing, $e$, are related and cannot be chosen independently.

This relationship implies that the center, "offset" wing support bearing may be placed at any location along the line that is tangent to the start of the arc curve and does not need to lie over the hinge in the bridge. This is important because there may be cases where the location of the offset bearing is constrained by the underlying support structure or other factors.

The geometric relationship described in Eqs. (5a) and (5b) is a direct result of the relationship between the wing's twist angle and the pitch angle of the joint. This quantity describes the amount of wing twist that occurs for a given pitch rotation of the bridge joint. Returning to Eq. (2) and noting that $w_{0} \approx L \theta$, where $\theta$ is the relative pitch angle between the two spans as shown in Fig. $6 \mathrm{~b}$ (assuming that $\theta \ll 1$ and $\varphi \ll 1)$ the two angles can be related by:

$\phi \approx \frac{L \theta}{2 e}$

or more conveniently

$\phi / \theta \approx L / 2 e$

This value is constant for the expected range of pitch rotations.

The resulting vertical curve radius will depend on the geometric parameters of the system and the pitch of the bridge joint. The radius of curvature is inversely related to the second derivative of the vertical displacement of the yoke curve. For a circular yoke curve, described by Eq. (6), this leads to an expression for the minimum vertical curve radius, located at the end of the CESuRa system $(x=0)$, of:

$$
\begin{aligned}
\min R(x) & \left.\approx\left(\frac{\mathrm{d}^{2} w(x)}{\mathrm{d} x^{2}}\right)^{-1}\right|_{x=0}=\left.\frac{-2 e}{w_{0}}\left(\frac{\mathrm{d}^{2} f(x)}{\mathrm{d} x^{2}}\right)^{-1}\right|_{x=0} \\
& =\left.\left(\frac{2 e}{\theta L}\right)\left(\frac{L^{3}}{r^{2}}\right)\left[\left(\frac{r}{L}\right)^{2}-\left(1-\frac{x}{L}\right)^{2}\right]^{3 / 2}\right|_{x=0} \\
& =r\left(\frac{2 e}{\theta L}\right)\left(1-\left(\frac{L}{r}\right)^{2}\right)^{3 / 2} .
\end{aligned}
$$

For the expected range of pitch rotations, the effective minimum radii of the vertical curve can be computed and compared to the applicable standard (e.g., $[18,19])$.

The three wing support bearings at A, B, and along the line $\mathrm{AC}$ are sufficient to support the wing. Because the wing rotates relative to the transition span about line $\mathrm{BC}$, a fourth bearing could be located along that line. However, three bearings are sufficient for equilibrium, and use of four renders the system statically indeterminate and the individual support reactions sensitive to thermally induced deformations and slight inaccuracies in installation. Thus, three bearings are recommended.

The location of the third offset support bearing along the line $\mathrm{AC}$ influences the wing geometry. Placing the bearing quite close to point $\mathrm{A}$ allows the wing to be relatively narrow and not to extend out to point $\mathrm{C}$. This is advantageous because the wing needs to be wide enough only to accommodate the bearings on it that support the bearer bars. A wing that is trapezoidal in plan and completely encompasses the bearing yoke constitutes a good choice and is shown schematically in Fig. 7. However, the convenience of a narrower wing comes at the price of increasing the bending moments in the wing and the forces in the bearings, so the designer will need to seek an appropriate compromise.

\subsection{Asymmetric Systems Subjected to Pitch}

To this point, the CESuRa system has been assumed to be symmetric, with the center of the system centered over the hinge joint between the floating and transition spans. If this is not possible and the system must be offset longitudinally from the joint centerline, the geometric requirements remain the same in principle but differ in detail. For example, it is no longer possible to achieve slope continuity at both ends of the CESuRa system using a symmetric yoke curve such as a second-order parabola or a circle. The designer may choose to use such a curve anyway, and accept the resulting discontinuity in slope at each end, or to adopt an asymmetric curve such as a cubic. An example of the latter is shown in Fig. 8a. However, if the mid-span of the CESuRa system is extremely eccentric with respect to the hinge joint, the yoke may lie outside the line connecting the bearings, as shown in Fig. 8b. This occurs if the centers of the joint and CESuRa system are more than $L / 3$ apart, where the wings are $2 L$ long measured along line $\mathrm{AB}$. The consequences are that the wings then

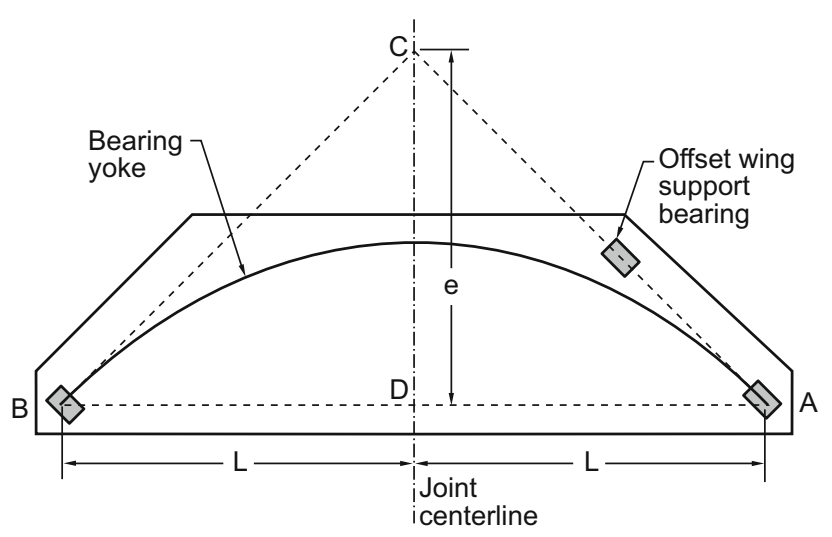

Fig. 7 Trapezoidal wing geometry and wing bearing placement 
(a)

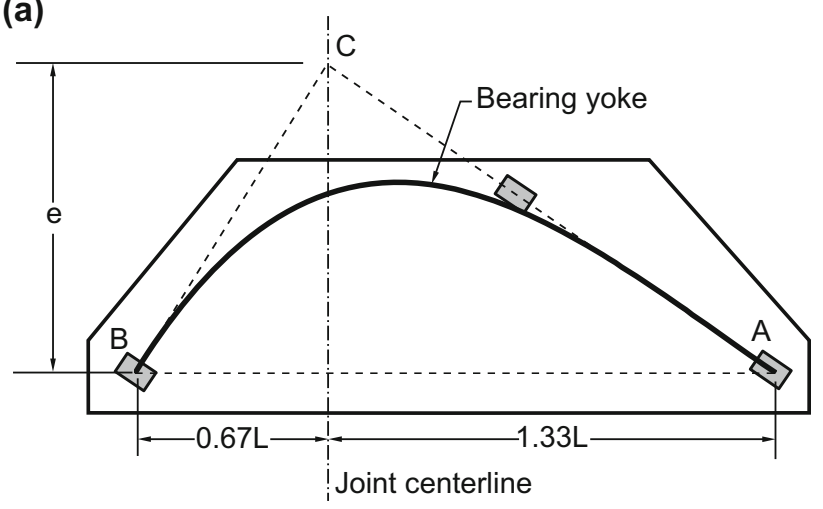

(b)

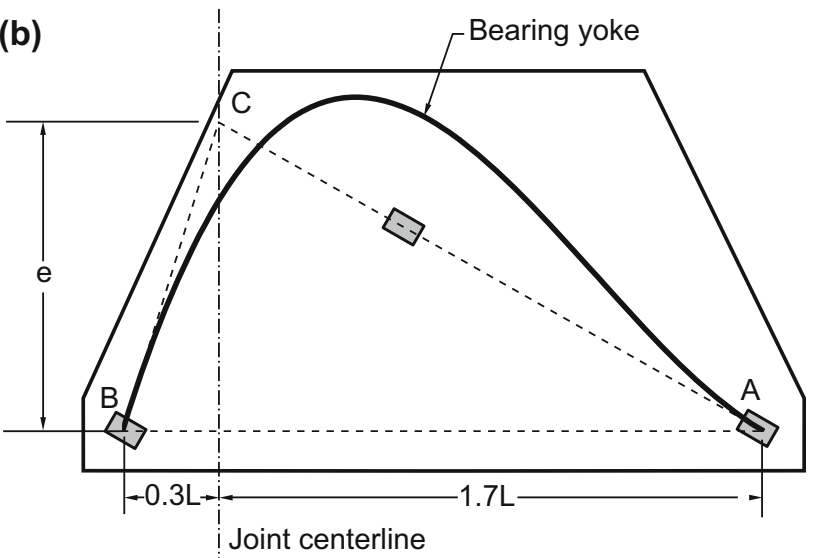

Fig. 8 Longitudinally offset CESuRa system, (a) $L / 3$ offset, (b) $7 \mathrm{~L} /$ 10 offset

need to be wider. That likely adds cost and may also reduce the wings' stiffnesses. The use of a symmetric system is therefore recommended wherever possible.

If, in a longitudinally offset system, a circular curve is still chosen for the yoke, small discontinuities in slope will exist at each end of the CESuRa system. To minimize the maximum error in such a case, the CESuRa system should be designed as if it were symmetrically placed and the offset wing support bearing should be moved parallel to the axis of the bridge joint to a position, $e_{\text {new }}$, given by:

$e_{\text {new }}=\frac{e}{1-L_{p} / L}$

where $e$ is the computed eccentricity given by Eq. (8), $L_{p}$ is the distance from the centerline of the wing to the centerline of the bridge joint, and $L$ is half of the length of wing. This procedure will modify the wing twist-to-pitch ratio described in Eq. (10) and ensure that the discontinuities at either end of the track bridge will be equal in magnitude and opposite in direction. The resulting errors in slope, $\pm L_{p} \theta / 2 L$, will be related to the pitch angle, the length of wing, and the size of the longitudinal offset. For practical applications, where the longitudinal offset is much smaller than the total length of the track bridge, this error is likely acceptably small in comparison with other errors, like the ones caused by the flexibility of the wings and their support bearings.

\subsection{Roll}

Minimizing discontinuities in the twist angle due to roll imposes a further condition on the system geometry. The dimensions of the system in the undeformed configuration are shown in Fig. 9a. Roll of the floating span will cause the end of one wing to rise and the other to fall, by equal distances, as shown in Fig. 9b.

For a symmetric system, assuming a counterclockwise roll rotation, the end of the wing that rests on the transition span (point B) falls through a distance $w_{0}$ to the position $\mathrm{B}^{*}$ and the end of the opposite wing (point B') rises through a distance $w_{0}$ to the position $\mathrm{B} *$. Point $\mathrm{D}$ falls to location $\mathrm{D}^{*}$, through a distance $w_{0} / 2$, so, and for point $\mathrm{C}$ to remain in contact with the floating span, the wing must rotate about axis $\mathrm{AB}^{*}$ through an angle $\phi$, given by Eq. (2). Point $\mathrm{D}^{\prime}$ rises to location $\mathrm{D}^{\prime} *$, through a distance $w_{0} / 2$, and the wing rotates about the axis $\mathrm{A}^{\prime} \mathrm{B}^{\prime} *$ to remain in contact with the floating span at point $C^{\prime}$. The total vertical displacement of a point along either of the bearing yokes consists of the displacement due to the pitch of the wing (either upward or downward) plus a component due to the rotation of the wing about either axis $\mathrm{AB}^{*}$ or axis $\mathrm{A}^{\prime} \mathrm{B}^{\prime *}$. The motion can be thought of as one side pitching up, and the other, down.

The vertical displacement of the rising rail is given by Eq. (3). The vertical displacement of the falling rail is given by Eq. (3) except the signs of the two terms are reversed. The distance between the rail centerlines is $2(s-f(x))$, so the roll angle, $\psi$, assuming that the roll angle is small (i.e., $\psi \ll 1$ ) at a location $\mathrm{x}$ on a horizontal plane is:

$\psi(x)=\frac{w_{0}}{2(s-f(x))}\left(\frac{x}{L}-\frac{f(x)}{e}\right)$

The rate of change of roll angle with respect to $\mathrm{x}$ is found by differentiating Eq. (13) with respect to $\mathrm{x}$ and is:

$$
\begin{array}{r}
\frac{\mathrm{d} \psi(x)}{\mathrm{d} x}=\frac{w_{0}}{2(s-f(x))^{2}}\{( \\
(s-f(x))\left(\frac{1}{L}-\frac{1}{e} \frac{\mathrm{d} f(x)}{\mathrm{d} x}\right) \\
\left.+\frac{\mathrm{d} f(x)}{\mathrm{d} x}\left(\frac{x}{L}-\frac{f(x)}{e}\right)\right\}
\end{array}
$$

For continuity of the rate of change of roll at the ends of the track bridge, $\frac{\mathrm{d} \psi(x)}{\mathrm{d} x}$ must be zero. At the end $x=0$, $f(0)=0$. Substitution in Eq. (14) leads to the requirement that $\left.\frac{\mathrm{d} f(x)}{\mathrm{d} x}\right|_{x=0}=e / L$. This is the same requirement that was found necessary for slope continuity under pitch loading. 
Fig. 9 Geometry for analysis of roll motions, (a) undeformed, (b) deformed (rotations are greatly exaggerated), (c) roll angle over a CESuRa system with a circular yoke curve
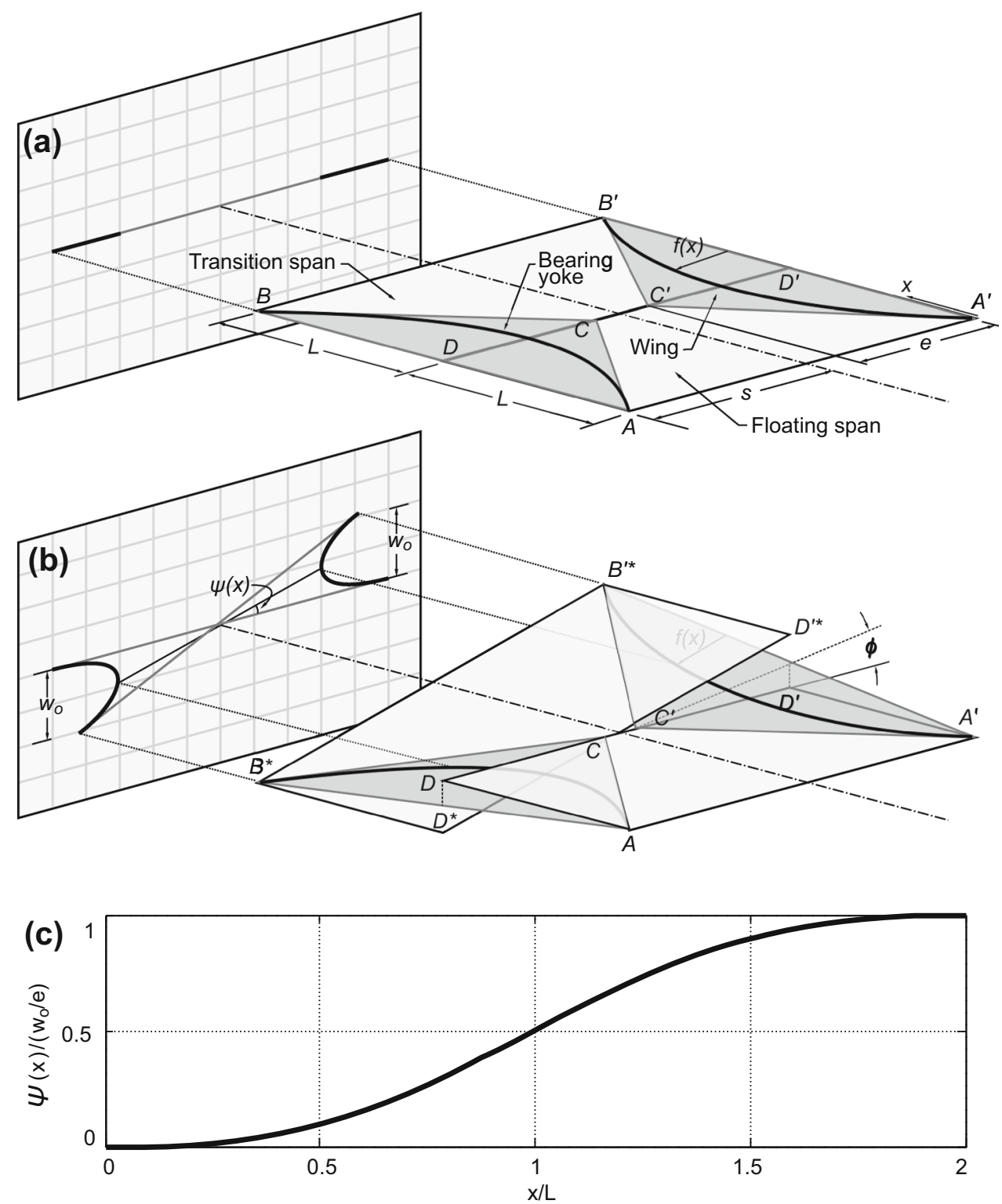

At the other end, $x=2 L, f(2 L)=0$, and setting $\frac{\mathrm{d} \psi(x)}{\mathrm{d} x}$ to zero leads to the requirement that $e=s$. The physical meaning is that the point $\mathrm{C}$, which defines the line $\mathrm{AC}$ on which the offset wing support bearing lies, must be located on the longitudinal centerline of the CESuRa system.

For a circular yoke curve or radius, $r$, as given by Eq. (6), Eq. (13) reduces to:

$$
\begin{aligned}
\psi(x)= & \frac{w_{0}}{2 e}\left[1-\left(1-\frac{x}{L}\right)\left(1-\frac{L}{e} \sqrt{\left(\frac{r}{L}\right)^{2}-\left(1-\frac{x}{L}\right)^{2}}\right.\right. \\
& \left.\left.-\frac{L}{e} \sqrt{\left(\frac{r}{L}\right)^{2}-1}\right)^{-1}\right]
\end{aligned}
$$

This sigmoid function is shown schematically in Fig. 9c for a system that satisfies Eq. (6).

\subsection{Vertical Acceleration}

The third criterion, namely minimization of the vertical acceleration of the vehicle, can be achieved by suitable selection of the yoke curve. The potential for achieving a significant increase in vehicle speed by using a CESuRa joint should also be considered [2]. The acceleration depends on the vertical curvature of the track and the vehicle properties such as mass, stiffness, and velocity. It is also affected by vehicle dimensions, such as the distance between axles within a bogie, and the distance between bogie centers. Because the vehicle properties may change during the lifetime of the track, only the track curvature is considered here. For a track vertical radius of curvature of $R$ and a vehicle speed of $v$, the vertical acceleration, $a_{\mathrm{w}}$, of the wheels is: 
$a_{\mathrm{w}}=\frac{v^{2}}{R}$

To minimize the peak curvature of the track on the bridge, the curvature should be the same everywhere, which leads to the selection of a parabolic yoke curve. In practice, a circular yoke curve would provide almost indistinguishable results. (Its peak curvature would be larger by a factor of $1+0.0004 \theta^{2}$, where $\theta$ is the rotation of the transition span in degrees.) Equation (16) could also be used to determine the range of operating speeds for the expected pitch rotations, given the vertical radius of curvature (Eq. 11) and the applicable vertical acceleration limits [e.g., 3, 19].

If the dynamic properties of the vehicle are taken into account, it is possible that some characteristic of the track geometry other than minimizing the peak curvature might control. For example, if arranging a smooth transition in acceleration from none at the ends of the track bridge to a maximum at mid-span were found to be critical, then this can be achieved by using a symmetric fourth-order polynomial. However, this arrangement would necessarily increase the peak acceleration of the wheels.

Practical factors such as the stiffness of the wings lead to movements under traffic which would momentarily change the vertical curvature, possibly negating the benefit of a more complex curve.

\subsection{Surge}

Surge displacements are longitudinal motions caused, for example, by expansion or contraction of the bridge. The CESuRa system plays no part in them; expansion joints in the rails on each end of each transition span are needed. The rails should be fixed to the deck at specific points along the span, between which they are free to expand and contract as a consequence of using fasteners that provide no longitudinal restraint. Thus, all the longitudinal motion of the rails is concentrated at the expansion joints. In the transition span, the rails can be anchored to the deck throughout its length. In the event of longitudinal motion of the bridge, the bridge deck, rather than just the rails, will experience longitudinal motion. Then, the wings of the CESuRa system will have to accommodate some longitudinal movement relative to the transition span. This displacement is accommodated at the guided bearing at one end of the wing.

\subsection{Yaw}

In the absence of pitch and roll, the response of the CESuRa system to yaw consists simply of in-plane deformation of the system of rails and bearer bars, acting as a frame; the rotations of the wings about their own longitudinal axes play no part. As the floating section of the bridge moves laterally, the transition span rotates as a rigid body about a vertical axis, so the track and the CESuRa at either end of it must accommodate that rotation. If the track lies at mid-width of the bridge, this involves lengthening of one rail and shortening of the other, but if, the tracks are at the side of the bridge, both rails must elongate or shorten, although by different amounts. The changes in length are accommodated by the expansion joints, in the same way as for surge.

Where there is significant yaw movement, part of the yaw rotation is manifested as a distributed curvature along the track bridge and part as concentrated rotations at the ends. The extent of each is governed by the lateral bending stiffness of the frame made up of the (longitudinal) rails and the (transverse) bearer bars. The fasteners that connect the rails to the bearer bars allow some rotation, and if fasteners with no longitudinal restraint are used, then the rotation is essentially free, so that system is flexible and promotes distributed curvature along the track bridge. Guard rails mounted rigidly in the track panel provide more frame action, make the track bridge stiffer, and thus force more of the total rotation into the concentrated rotations at the ends of the track bridge.

In the absence of pitch or roll rotations, which would require a CESuRa system in the horizontal plane, a CESuRa system could, instead, be installed in the vertical plane, as shown schematically in Fig. 10, to address yaw rotation about the vertical axis. The problem is in principle the same as the one in the vertical plane that the CESuRa was developed to solve; the underlying bridge undergoes a concentrated rotation, but that of the rails must be distributed.

The governing geometric relationships are the same as for a pitch rotation in the horizontal plane. The deformed

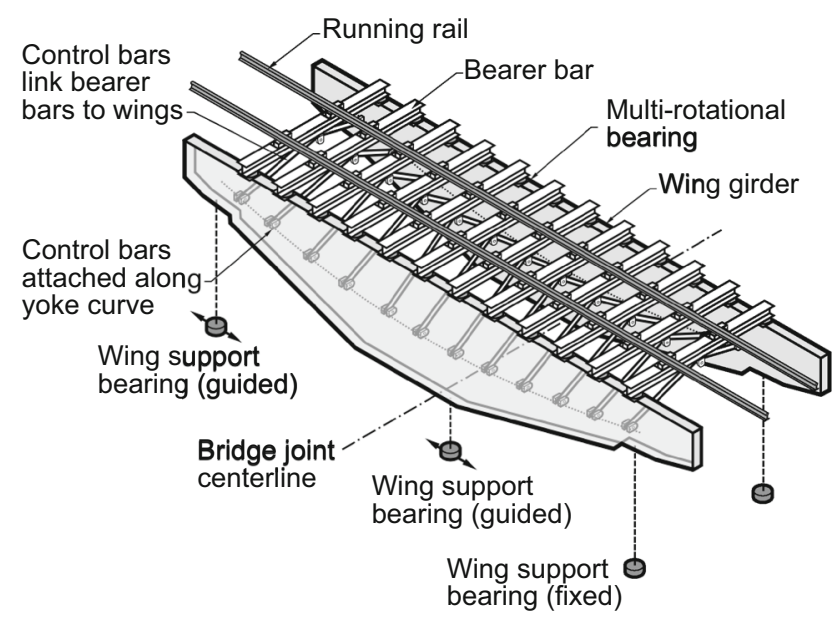

Fig. 10 CESuRa system in the vertical plane 
Fig. 11 Geometry for analysis of yaw rotations

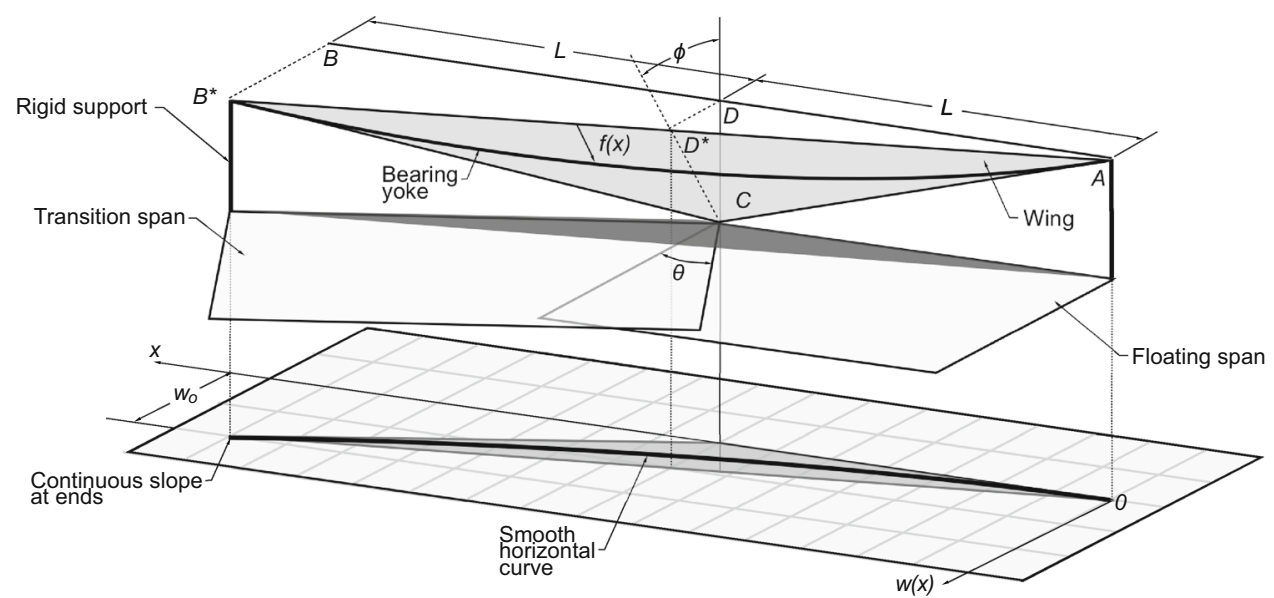

two tracks in the middle of the CESuRa track panels, could be employed, as shown in Fig. 12.

\section{Bearing Requirements}

Two primary bearing types are needed: three wing support bearings per wing, and a number of multi-rotational bearings along the bearing yoke for the bearer bars that support the rails. In each case, the bearings need to accommodate motion in several directions.

For the wing support bearings, vertical stiffness is desirable to prevent excessive deflection of the bearing and the corresponding bending of the rails. If a bearing type is selected that has different rotation capacities about different axes, the bearing should be oriented so that its largest capacity is available for rotation about the axis $\mathrm{AC}$ in Fig. 6, since that is the axis of applied rotation. Elastomeric bearings often provide the most economical support system and are particularly attractive when the primary axes of rotation are unclear, such as in a skewed or curved bridge. However, their vertical and rotational stiffnesses are linked, in that a high vertical stiffness requires a high shape factor, which in turn leads to a high rotational stiffness. Thus, in cases where large rotations need to be accommodated while maintaining high vertical stiffness, some other bearing type may be needed.

The bearer bar support bearings must allow for rotation about all three axes, with the largest motions likely about the longitudinal axis of the bridge. They must also accommodate some transverse displacement, which occurs as the wings rotate. Although this displacement is nominally a second-order effect, it is nonetheless large enough that provision must be made for it.

Fig. 12 Combined horizontal and vertical CESuRa systems 


\section{Application of the CESuRa System}

The expected maximum rotations for the pontoon bridge in Seattle, WA, USA, are $2.2^{\circ}$ in pitch and $1.1^{\circ}$ in yaw [8]. The estimated roll demand on the CESuRa system is small, because, when the floating span rolls, most of the rotation is manifested in the twisting of the transition span structure. The CESuRa track bridge rests on the transition span at a distance of approximately $\mathrm{L}$ from the bridge joint, as shown in Fig. 9, so the roll rotation that it needs to accommodate is only approximately $\theta_{\text {roll }} * L / L_{\mathrm{ts}}$, where $L_{\mathrm{ts}}$ is the length of the transition span, $2 L$ is the length of the track bridge and $\theta_{\text {roll }}$ is the roll rotation of the floating span. In practice, $L \ll L_{\mathrm{ts}}$, so the roll demand on the CESuRa system is small compared with the pitch demand.

The geometry of the CESuRa system for the floating bridge application is shown in Fig. 13. Two different configurations for the wing support bearing were used at either end of the transitions span, the "interior" and "exterior" joints, due to structural considerations of the underlying supports. The wings were steel box girders that

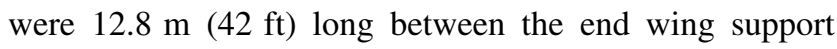
bearings, stiffened by an upturned edge beam, and supported by elastomeric bridge bearings. One end bearing was fixed, and the other was guided to move only in the longitudinal direction. The center bearings were offset $1.52 \mathrm{~m}$ and $0.91 \mathrm{~m}$ ( 5 and $3 \mathrm{ft}$ ) from the centerline of the wings for the exterior and interior CESuRa systems, respectively, and $0.97 \mathrm{~m}(3.17 \mathrm{ft})$ inboard from a longitudinal line connecting the end wing support bearings. The yokes were circular with radii of $42.7 \mathrm{~m}$ (140 ft).

Seventeen bearer bars supported the rail, spaced at $0.7 \mathrm{~m}$ (27 in.). The rails were connected to the bearer bars using elastomeric fasteners with clips that provide zero longitudinal restraint. Friction pendulum system (FPS) bearings were used to support the bearer bars. The FPS bearings were specially designed to limit uplift of the bearer bars, and primarily accommodated translation and rotation in the direction perpendicular to the rails, although some limited amount of movement was permitted by the bearings in all six degrees of freedom.

The height of the track above the wing support bearings (due to the height of the wing girders, the FPS bearings, bearer bars, and rail fasteners) modifies the kinematics of the idealized system described previously. The twist of the wing girders leads to an additional vertical displacement of the yoke curves caused by the rigid body rotation of the wing girder, and the yoke curves translate toward or away from one another, depending on the direction of twist. A similar coupling of rotation and vertical movement occurs at the FPS bearings, due to their curved sliding interfaces. These can both be considered second-order effects and were minimized in the floating bridge application by limiting the height of the components of the system. This was achieved by using a thin wing girder along the bearing yoke surface that was stiffened by an edge beam and coping the ends of the bearer bars where they connected to the friction pendulum bearings.
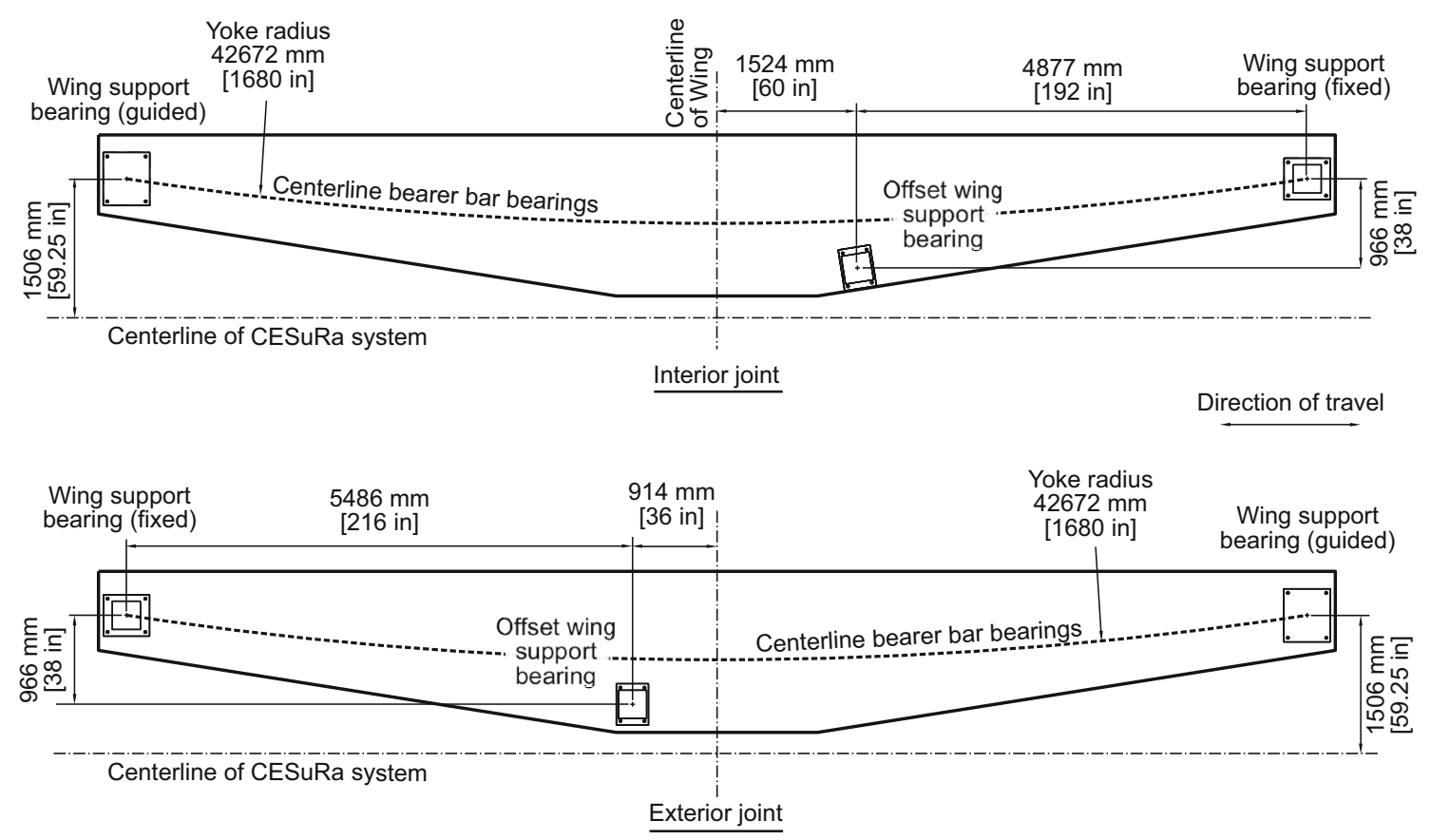

Fig. 13 Wing Geometry used on the Homer M. Hadley Bridge 
Guard rails were installed over the length of the track bridge to prevent wheel derailment. The addition of the guard rails also had the effect of stiffening the frame made up of the rails and bearer bars, thereby limiting lateral movement of the rails under train passage. The guard rails were continuous across the CESuRa system, connected to the bearer bars using machined pins, and connected to the guard rail of the adjacent span through a sliding mechanism. These provisions were meant to limit the frame action contribution of the guard rails in order to promote distributed curvature of the track-rail frame under yaw movements while also accommodating surge movements.

The CESuRa system was tested in three phases. First, proof-of-concept testing was carried out at the University of Washington, using a part of the track bridge constructed at full scale. The primary purpose was to determine whether the desired motions could be achieved and the necessary forces resisted $[14,15]$. Then, two complete track bridges were tested at the fabrication facilities of Jesse Engineering, in Tacoma, WA, USA, after they had been constructed, to verify that the complete system would behave as planned, particularly in view of the tight tolerance requirements that were identified during the first phase of testing. Last, the fabricated track bridges were shipped as complete units to Pueblo, CO, USA, where they were installed in the test track at the Transportation Technology Center [16, 17]. They were configured in various combinations of pitch, roll, and yaw, and trains were run over them at design speeds in order to investigate the dynamic response. The results demonstrated that the system performed as desired in terms of rider comfort and safety and that the geometric concepts underlying the accommodation of pitch functioned as intended [16, 17].

\section{Conclusions}

The following conclusions can be drawn from the investigation:

1. The CESuRa concept provides the track with the ability to undergo pitch and roll motions without risk of damage to the rails, while maintaining full vertical support of the track across the bridge joint. The same principle, with the physical mechanism rotated through $90^{\circ}$, could also be used to accommodate yaw rotations, although using both systems simultaneously is likely impractical. Other motions, such as longitudinal expansion, can and should be addressed separately.

2. The system is a 3-D assembly of parts that undergo rigid body motions to accommodate the necessary bridge displacements. The system is most easily understood assuming small joint rotations; however, the system is not limited to small deformations. The only element that must deform is the rail, and a larger rotation will require a longer CESuRa assembly in order to limit the rail strains to an acceptable level.

3. The shape of the yoke can be tuned to achieve specific characteristics, such as minimizing the peak acceleration. If the system is optimized in this way, the dynamic characteristics of the vehicle should be accounted for in the process.

4. The height of the track above the support bearings introduces small discontinuities in the slope of the rails at the ends of the CESuRa assembly. The use of lowerprofile wings and bearer bars reduces those discontinuities, but their lower stiffness increases deflections due to the passage of rail vehicles. Therefore, there is a balance to be drawn.

5. The performance of the system depends on achieving on site the geometry selected during design. The tight tolerances required may best be realized by building the CESuRa system in the fabrication shop and shipping it to site as a single unit.

Acknowledgements The authors would like to acknowledge industry partners on this project; Tom Cooper and John Harrison from WSP; Bryan Williams from Stacy and Witbeck, Inc who worked for Jacobs Engineering during the design and testing of the track bridges, and John Sleavin from Sound Transit.

Open Access This article is distributed under the terms of the Creative Commons Attribution 4.0 International License (http://crea tivecommons.org/licenses/by/4.0/), which permits unrestricted use, distribution, and reproduction in any medium, provided you give appropriate credit to the original author(s) and the source, provide a link to the Creative Commons license, and indicate if changes were made.

Funding The application of the CESuRa concept and the subsequent testing was funded by Sound Transit through a contract with Parsons Brinckerhoff with Grants from the US Federal Transit Administration, which support the project. The findings and conclusions contained herein are those of the authors alone.

\section{Compliance with Ethical Standards}

Conflict of interest The authors declare that they have no conflict of interest.

\section{References}

1. KPFF (2005) Homer Hadley interstate 90 Floating Bridge test program for light rail transit draft test report

2. Foan AR, Cooper T (2015) A CESuRa in the Track. J Perm Way Inst 133(1):8-13

3. UIC (2009) UIC 776-2 design requirements for rail-bridges based on interaction phenomena between train, track, and bridge 
4. Armitage RA, Sharpe P, Heggie W, Rogers A (2002) Innovative design of transition zones. In: Proceedings, railway engineering 2002: 5th international conference and exhibition, London, UK, p 23

5. Gallage C, Dareeju B, Dhanasekar M (2013) State-of-the-art: track degradation at bridge transitions. In: Proceedings, 4th international conference on structural engineering and construction management, Kandy, Sri Lanka, pp 40-52

6. Sañudo R, dell'Olio L, Casado JA, Carrascal IA, Diego S (2016) Track transitions in railways: a review. Constr Build Mater 112:140-157

7. Wang H, Markine V (2018) Corrective countermeasure for track transition zones in railways: adjustable fastener. Eng Struct 169:p1-p14

8. Quade PB (2006) Douglas Inc. East Corridor HCT-Summary of I-90 Floating Bridge (Homer Hadley) Studies. In: Final report for sound transit

9. Rao RR, Sanghvi S (2000) Design of continuous welded rail on a suspension bridge. In: Proceedings, AREMA annual conference, Dallas, TX, USA

10. Hess J (2009) Rail expansion joints-the underestimated track work materials? In: Track-bridge interaction on high-speed railways, CRC Press, London, pp 149-164

11. Cooper T, Foan A, Thonstad T (2013) I-90 track bridge-the challenge of taking light rail vehicles on to the Homer Hadley floating bridge. In: Proceedings, international bridge conference, Pittsburgh, PA, USA

12. Cooper T, Sleavin J, Foan A, Thonstad T (2014) Unprecedented connections. Civ Eng 84:56-63

13. Sedarat H, Talebinejad I, Kozak A, Krimotat A, Cooper T, Harrison JA, Sleavin J, Cornish P (2013) Dynamic response of a floating bridge to a moving light rail train. In: Proceedings, structures congress 2013, Pittsburg, PA, USA

14. Thonstad T (2013) Specialized track bridge structure for light rail construction on floating bridges. In: M.S.C.E. Thesis, University of Washington, Seattle

15. Thonstad T, Stanton JF, Foan A, Sleavin J (2016) How to put light rail on a floating bridge. Transp Res Rec 2545:46-55

16. Ketchum C, Cooper T, Foan A, Joy R, Sedarat H, Sleavin J (2015) Dynamic simulations in support of installation of light rail tracks on the Homer H. Hadley Memorial floating bridge. In: Proceedings, the Stephenson conference, London, UK

17. Harrison JA, Cooper TR, Lawrence L, Williams B, Ketchum CD, Sleavin J (2017) Integration of light rail on the I-90 floating bridge across lake Washington. Transp Res Rec 2607:p74-p81

18. RSSB (2011) GC/RT5021 Iss 5: track system requirements. Rail safety and standards board, London, UK

19. AREMA (2002) Manual for railway engineering. American Railway engineering and maintenance of way association, Lanham, MD, USA 